\title{
RAMSEY THEORY IN NONCOMMUTATIVE SEMIGROUPS
}

\author{
VITALY BERGELSON AND NEIL HINDMAN
}

\begin{abstract}
By utilizing ultrafilters we give a general version of the Central Sets Theorem [6, Proposition 8.21]. This enables us to derive noncommutative versions of van der Waerden's Theorem and several of its generalizations. We also derive some standard results, including the Hales-Jewett Theorem.
\end{abstract}

\section{INTRODUCTION}

Van der Waerden's Theorem [15] says that whenever the set $\mathbb{N}$ of positive integers is divided into finitely many classes, some one of these classes contains arbitrarily long arithmetic progressions. The assertion makes sense in any semigroup $(S, \cdot)$, where the arithmetic progression $\{a, a+d, a+2 d, \ldots, a+l d\}$ becomes, when written multiplicatively, $\left\{a, a d, a d^{2}, \ldots, a d^{l}\right\}$. Moreover the assertion is true in this context as a trivial consequence of van der Waerden's Theorem itself. (Given $x \in S$ and $l \in N$ choose $b$ and $c$ in $\mathbb{N}$ with $\left\{x^{b}, x^{b+c}, x^{b+2 c}, \ldots, x^{b+l c}\right\}$ contained in one class. Let $a=x^{b}$ and $d=x^{c}$.)

However when one deals with general semigroups there are at least two drawbacks to such a version of van der Waerden's Theorem. In the first place there is no guarantee in general that the "increment" $d$ will not be an identity for $S$. In the second place even if the "increment" of the progression $\left\{x^{b}, x^{b+c}, \ldots, x^{b+l c}\right\}$ is nontrivial there is no guarantee that the members $x^{b+i c}$ are all distinct for different $i$.

As for the first drawback, there is a strengthened version of van der Waerden's Theorem which allows us to at least attempt to avoid such trivialities. Given any infinite subset $D$ of a right cancellative semigroup, there is a sequence $\left\langle x_{n}\right\rangle_{n=1}^{\infty}$ in $D$ all of whose decreasing finite products are distinct. (The decreasing finite products are all expressions of the form $x_{m(k)} \cdot x_{m(k-1)} \cdots x_{m(1)}$ with $m(k)>m(k-1)>\cdots>m(1)$. One could of course work equally well with the increasing finite products.) We write $\operatorname{FP}\left(\left\langle x_{n}\right\rangle_{n=1}^{\infty}\right)$ for the set of all decreasing finite products of $\left\langle x_{n}\right\rangle_{n=1}^{\infty}$. Similarly in an additively written semigroup $\operatorname{FS}\left(\left\langle x_{n}\right\rangle_{n=1}^{\infty}\right)$ is the set of (decreasing) finite sums.

The following theorem is a consequence of the Hales-Jewett Theorem. (See Theorem 1.3 below. See also [7].)

Received by the editors January 22, 1990.

1980 Mathematics Subject Classification (1985 Revision). Primary 05A17; Secondary 22A15.

Key words and phrases. Ramsey theory, noncommutative semigroups, van der Waerden's Theorem.

The authors gratefully acknowledge support received from the National Science Foundation via grants DMS-8700842 and DMS-8901058 respectively. 
1.1. Theorem. Let $\left\langle y_{n}\right\rangle_{n=1}^{\infty}$ be a sequence in $\mathbb{N}$ and let $l \in \mathbb{N}$. Whenever $\mathbb{N}$ is divided into finitely many classes, some one class contains $\{a, a+d, \ldots, a+l d\}$ with $a \in \mathbb{N}$ and $d \in \mathrm{FS}\left(\left\langle y_{n}\right\rangle_{n=1}^{\infty}\right)$.

Among the results of the present paper is the fact that the natural analogue of Theorem 1.1 holds in any commutative semigroup. Unfortunately, this analogue may fail in a noncommutative semigroup. For example, let $S$ be the free semigroup on the distinct generators $y_{1}, y_{2}, y_{3}, \ldots$. (That is $S$ is the set of words on the alphabet $\left\{y_{1}, y_{2}, \ldots\right\}$ with concatenation as the operation.) Given $w \in S$, let $\varphi(w)$ count the number of occurrences of letters $y_{m} y_{n}$ adjacent in $w$ with $m \leq n$. (Thus $\varphi\left(y_{6} y_{4} y_{5} y_{3} y_{3} y_{4}\right)=3$.) Let $A_{1}=\{w \in$ $S: \varphi(w)$ is odd $\}$ and let $A_{2}=\{w \in S: \varphi(w)$ is even $\}$. Given any $w \in S$ and $d \in \operatorname{FP}\left(\left\langle y_{n}\right\rangle_{n=1}^{\infty}\right)$ one has $\varphi\left(w d^{2}\right)=\varphi(w d)+1$ so neither $A_{1}$ nor $A_{2}$ contains $\left\{w d, w d^{2}\right\}$. The affirmative result (for commutative semigroups) is none the less a special case of a noncommutative result in which the $t$ th term of the progression has the form $a_{n+1} d_{m(n)}^{t} a_{n} d_{m(n-1)}^{t} a_{n-1} \cdots a_{2} d_{m(1)}^{t} a_{1}$. (See Corollary 3.1.)

As to the second drawback, let $p \in \mathbb{N}$ and let $G$ be the infinite direct sum of cyclic groups of order $p$. It is clear that if $l$ is bigger than $p$, elements of the progression $\left\{x^{b}, x^{b+c}, \ldots, x^{b+l c}\right\}$ cannot all be distinct. The following theorem (which is a special case of the Graham-Leeb-Rothschild Theorem [9]) however, provides us with a quite satisfactory analogue of van der Waerden's Theorem for such groups.

1.2. Theorem. Let $V$ be an infinite-dimensional vector space over a finite field $F$. Whenever $V$ is partitioned into finitely many classes, some one class contains affine subspaces of arbitrarily high dimension.

Now both Theorems 1.1 and 1.2 are consequences of the quite general combinatorial theorem dealing with free semigroups, the Hales-Jewett Theorem.

1.3. Theorem (Hales-Jewett [10]). Let $S$ be the free semigroup over a finite set $\Omega$ and let $x$ be a variable not in $\Omega$. Let $S$ be partitioned into finitely many classes. There is a word $\tau(x)$ over $\Omega \cup\{x\}$ in which $x$ actually occurs such that $\{\tau(a): a \in \Omega\}$ is contained in one class, where $\tau(a)$ is $\tau(x)$ with all occurrences of $x$ replaced by $a$.

To see that Theorem 1.1 follows from Theorem 1.3, let $\left\langle y_{n}\right\rangle_{n=1}^{\infty}$ be a sequence in $\mathbb{N}$, let $l, r \in \mathbb{N}$ and assume $\mathbb{N}=\bigcup_{j=1}^{r} A_{j}$. Let $\Omega=\{0,1, \ldots, l\}$ and let $S$ be the free semigroup over $\Omega$. Given $w=a_{1} a_{2} \cdots a_{t}$ in $S$ (with each $a_{i} \in \Omega$ ) let $\varphi(w)=1+\sum_{i=1}^{t} a_{i} y_{i}$. (We add the 1 to be sure $\varphi(w) \in \mathbb{N}$.) For $j \in\{1,2, \ldots, r\}$ let $B_{j}=\left\{w \in S: \varphi(w) \in A_{j}\right\}$. Choose $k \in\{1,2, \ldots, r\}$ and $\tau(x)=a_{1} a_{2} \cdots a_{t}$ where at least one $a_{i}=x$ with $\{\tau(a): a \in \Omega\} \subseteq B_{k}$. Let $F=\left\{i \in\{1,2, \ldots, t\}: a_{i}=x\right\}$ and let $G=\{1,2, \ldots, t\} \backslash F$. Let $d=$ $\sum_{i \in F} y_{i}$ and let $b=1+\sum_{i \in G} a_{i} y_{i}$ (with $b=1$ if $G=\varnothing$ ). Then given $a \in \Omega$, $b+a d=\varphi(\tau(a))$ so $\{b, b+d, \ldots, b+l d\} \subseteq A_{k}$.

We illustrate how Theorem 1.2 can be derived from Theorem 1.3 by letting $F=\{0,1,2\}$, the field with three elements, partitioning $V=\bigcup_{i=1}^{r} A_{i}$ into finitely many pieces, and showing how to obtain a two-dimensional subspace in one piece. Let $B$ be a basis for $V$ and let $\left\langle\vec{y}_{n}\right\rangle_{n=1}^{\infty}$ and $\left\langle\vec{z}_{n}\right\rangle_{n=1}^{\infty}$ be disjoint sequences in $B$. Let $\Omega=\{(0,0),(0,1),(0,2),(1,0),(1,1),(1,2),(2,0)$, 
$(2,1),(2,2)\}$. For $a \in \Omega$ let $\pi_{1}(a)$ and $\pi_{2}(a)$ be the first and second coordinates of $a$. For $w=a_{1} a_{2} \cdots a_{t}$ in $S$, let $\varphi(w)=\sum_{i=1}^{t}\left(\pi_{1}\left(a_{i}\right) \cdot \vec{y}_{i}+\pi_{2}\left(a_{i}\right) \cdot \vec{z}_{i}\right)$ and for $j \in\{1,2, \ldots, r\}$ let $B_{j}=\left\{w \in S: \varphi(w) \in A_{j}\right\}$. Choose $k \in$ $\{1,2, \ldots, r\}$ and $\tau(x)=a_{1} a_{2} \cdots a_{t}$ (again with some $a_{i}=x$ ) with $\{\tau(a): a \in$ $\Omega\} \subseteq B_{k}$. Let $F=\left\{i \in\{1,2, \ldots, t\}: a_{i}=x\right\}$, and let $G=\{1,2, \ldots, t\} \backslash F$. Let $\vec{b}=\sum_{i \in G}\left(\pi_{1}\left(a_{i}\right) \cdot \vec{y}_{i}+\pi_{2}\left(a_{i}\right) \cdot \vec{z}_{i}\right)$, with $\vec{b}=\overrightarrow{0}$ if $G=\varnothing$. Let $\vec{u}=\sum_{i \in F} \vec{y}_{i}$, let $\vec{v}=\sum_{i \in F} \vec{z}_{i}$, and let $W$ be the subspace of $V$ generated by $\{\vec{u}, \vec{v}\}$. Then $\vec{b}+W=\{\varphi(\tau(a)): a \in \Omega\} \subseteq A_{k}$.

Most of our results are obtained from a generalization to arbitrary semigroups of the Central Sets Theorem [6, Proposition 8.21]. This generalization is simultaneously a generalization of the Hales-Jewett Theorem. To give an idea of the flavor of our results, we quote here one of the consequences; Corollary 3.1.

3.1. Corollary (Noncommutative van der Waerden). Let $S$ be any semigroup, let $l, r \in \mathbb{N}$, let $\left\langle d_{m}\right\rangle_{m=1}^{\infty}$ be a sequence in $S$, and let $S=\bigcup_{k=1}^{r} A_{k}$. There exist $k \in\{1,2, \ldots, r\}, n \in \mathbb{N}, a_{1}, a_{2}, \ldots, a_{n+1} \in S$, and $m(1)<m(2)$ $<\cdots<m(n)$ in $\mathbb{N}$ with $\left\{a_{n+1} a_{n} \cdots a_{2} a_{1}, a_{n+1} d_{m(n)} a_{n} d_{m(n-1)} \cdots a_{2} d_{m(1)} a_{1}\right.$, $\left.a_{n+1} d_{m(n)}^{2} a_{n} d_{m(n-1)}^{2} \cdots a_{2} d_{m(1)}^{2} a_{1}, \ldots, a_{n=1} d_{m(n)}^{l} a_{n} d_{m(n-1)}^{l} \cdots a_{2} d_{m(1)}^{l} a_{1}\right\} \subseteq A_{k}$. If $S$ is cancellative with $a 1$ and for each $i \in\{1,2, \ldots, l\}$ the sequence $\left\langle d_{m}^{i}\right\rangle_{m=1}^{\infty}$ is one-to-one, then the $l+1$ entries can be chosen to be distinct.

We establish our generalization of the Central Sets Theorem in $\S 2$. (We had established in [2] a generalization valid in certain commutative semigroups, which we called prenatural. It is interesting that the proof of the more general result presented here is much simpler.)

In $\S 3$ we derive several consequences of the main result including a multidimensional version of the noncommutative van der Waerden Theorem, that is a noncommutative version of Gallai's Theorem. (See [13].)

\section{Generalizing the CENTRAL SETS THEOREM}

The proof of our main result utilizes an idea of Furstenberg and Katznelson developed in the context of enveloping semigroups. See [1] for an elementary derivation of most facets of this idea in the context of the Stone-Čech compactification, which we use here.

Given a discrete semigroup $(S)$, we take the Stone-Čech compactification $\beta S$ of $S$ to be $\{p: p$ is an ultrafilter on $S\}$, identifying the points of $S$ with the principal ultrafilters. (An ultrafilter $p$ on $S$ may be thought of as a $\{0,1\}$ valued finitely additive measure $\mu_{p}$ on $P(S)$. The statements $A \in p$ and $\mu_{p}(A)=1$ are synonymous.) Given $A \subseteq S$, one lets $\bar{A}=\{p \in \beta S: A \in p\}$. Then in fact $\bar{A}=\operatorname{cl}_{\beta S} A$ and $\{\bar{A}: A \subseteq S\}$ forms a basis for the closed sets of $\beta S$. But the fact that we care about is that $\{\bar{A}: A \subseteq S\}$ forms a basis for the open sets of $\beta S$ as well. (See [11] for an elementary derivation of the basic facts about the Stone-Čech compactification.)

The operation - extends to $\beta S$ making $(\beta S, \cdot)$ a compact left topological semigroup. That is, for each $p \in \beta S$ the function $\lambda_{p}: \beta S \rightarrow \beta S$ defined by $\lambda_{p}(q)=p \cdot q$ is continuous. In addition, if $x \in S$, then the function $\rho_{x}: \beta S \rightarrow$ $\beta S$ defined by $\rho_{x}(p)=p \cdot x$ is continuous. The operation is characterized in the following way. Given $p, q \in \beta S$ and $A \subseteq S$, one has $A \in p \cdot q$ if and only 
if $\{x \in S: A / x \in p\} \in q$, where $A / x=\{y \in S: y \cdot x \in A\}$. (For an elementary derivation of the basic facts about $(\beta S, \cdot)$ see [12].)

A nonempty subset $B$ of a semigroup $S$ is a right ideal provided $B \cdot S \subseteq B$, a left ideal provided $S \cdot B \subseteq B$, and an ideal if it is both a left and a right ideal.

2.1. Lemma. Let $S$ be a compact Hausdorff left topological semigroup. Each right ideal of $S$ contains a minimal right ideal and each minimal right ideal is closed.

Proof. This is a routine Zorn's Lemma argument. (Or see [1].)

We now define several specialized notions used in our main proofs. Recall that we write our products in decreasing order of indices. Thus if $f:\{2,3,7\} \rightarrow$ $\{0,1,2,3\}$ with $f(2)=3, f(3)=1$, and $f(7)=2$, then $\prod_{n \in\{2,3,7\}} y_{f(n), n}$ $=y_{2,7} y_{1,3} y_{3,2}$.

The terms defined below depend on the choice of $S, l$, and the sequences $\left\langle y_{i, n}\right\rangle_{n=1}^{\infty}$, but we suppress reference to this dependence in the definition and in the lemmas that follow.

2.2. Definition. Let $S$ be a semigroup, let $l \in \mathbb{N}$, and for each $i \in\{0,1, \ldots, l\}$ let $\left\langle y_{i, n}\right\rangle_{n=1}^{\infty}$ be a sequence in $S$.

(a) Given $k \in \mathbb{N}, P_{k}=\left\{\prod_{n \in F} y_{f(n), n}: F\right.$ is a finite nonempty subset of $\mathbb{N}$ with $\min F \geq k$ and $f: F \rightarrow\{0,1, \ldots, l\}$.

(b) Given $a \in P_{1}, \mathscr{S}(a)=\{F: F$ is a finite nonempty subset of $\mathbb{N}$ and there exists $f: F \rightarrow\{0,1, \ldots, l\}$ with $\left.a=\prod_{n \in F} y_{f(n), n}\right\}$.

(c) $X=\bigcap_{k=1}^{\infty} \bar{P}_{k}$.

(d) Given $k \in \mathbb{N}, I_{k}=\left\{\left(a_{n+1} a_{n} \cdots a_{2} a_{1}, a_{n+1} y_{1, m(n)} a_{n} \cdots a_{2} y_{1, m(1)} a_{1}, \ldots\right.\right.$, $\left.a_{n+1} y_{l, m(n)} a_{n} \cdots a_{2} y_{l, m(1)} a_{1}\right):$ there exist $F_{1}, F_{2}, \ldots, F_{n+1}$ with each $a_{j} \in P_{1}$ and each $F_{j} \in \mathscr{S}\left(a_{j}\right)$ and $\min F_{n+1}>m(n)>\max F_{n} \geq \cdots>\max F_{2} \geq$ $\left.\min F_{2}>m(1)>\max F_{1} \geq \min F_{1} \geq k\right\}$.

(e) Given $k \in \mathbb{N}, E_{k}=I_{k} \cup\left\{(a, a, \ldots, a) \in S^{l+1}: a \in P_{k}\right\}$.

(f) $T=(\beta S)^{l+1}$.

(g) $I=\bigcap_{k=1}^{\infty} \mathrm{cl}_{T} I_{k}$.

(h) $E=\bigcap_{k=1}^{\infty} \mathrm{cl}_{T} E_{k}$.

As an example, let $l=3$ and let $a=y_{3,7} y_{0,5} y_{2,4} y_{1,2}$. Then $a \in P_{2}$ (with $f(7)=3, f(5)=0, f(4)=2$, and $f(2)=1)$ and $\{2,4,5,7\} \in \mathscr{S}(a)$. On the other hand, if $b=y_{2,4} y_{0,5} y_{1,2} y_{3,7}$ then $b$ is not (on its face) in $P_{2}$ since the second indices are not decreasing.

2.3. Lemma. $X$ is a compact subsemigroup of $\beta S$.

Proof. Trivially $X$ is nonempty and compact. Let $p, q \in X$. To see that $p \cdot q \in X$, let $k \in \mathbb{N}$ be given and let $U$ be an open neighborhood of $p \cdot q$. We need to show $U \cap P_{k} \neq \varnothing$. Since $\lambda_{p}$ is continuous, pick a neighborhood $V$ of $q$ with $p \cdot V \subseteq U$. Since $V$ is a neighborhood of $q$, pick $a \in V \cap P_{k}$, pick $F \in \mathscr{S}(a)$ with $\min F \geq k$, and pick $f: F \rightarrow\{0,1, \ldots, l\}$ such that $a=\prod_{n \in F} y_{f(n), n}$. Let $m=\max F+1$. Now $p \cdot a \in U$ and $a \in S$ so, by the continuity of $\rho_{a}$, pick a neighborhood $W$ of $p$ with $W \cdot a \subseteq U$. Pick $b \in W \cap P_{m}$, pick $G \in \mathscr{S}(b)$ with $\min G \geq m$, and pick $g: G \rightarrow\{0,1, \ldots, l\}$ such that $b=\prod_{n \in G} y_{g(n), n}$. Let $H=F \cup G$ and let $h=f \cup g$. Then $b \cdot a=\prod_{n \in H} y_{h(n), n}$ so $b \cdot a \in U \cap P_{k}$. 
2.4. Lemma. $E \subseteq X^{l+1}$.

Proof. Given $k \in \mathbb{N}$ we have immediately that $E_{k} \subseteq\left(P_{k}\right)^{l+1}$ and hence $\mathrm{cl}_{T} E_{k} \subseteq\left(\bar{P}_{k}\right)^{l+1}$.

2.5. Lemma. $E$ is a subsemigroup of $X^{l+1}$ and $I$ is an ideal of $E$.

Proof. Let $\vec{p}, \vec{q} \in E$. We show that $\vec{p} \cdot \vec{q} \in E$ and if either $\vec{p} \in I$ or $\vec{q} \in I$, then $\vec{p} \cdot \vec{q} \in I$. Let $k \in N$ and let $U$ be an open neighborhood of $\vec{p} \cdot \vec{q}$. We show that $U \cap E_{k} \neq \varnothing$ and that if either $\vec{p} \in I$ or $\vec{q} \in I$ then $U \cap I_{k} \neq \varnothing$. To do this we repeatedly utilize appropriate continuity conditions.

Pick a neighborhood $V$ of $\vec{q}$ with $\vec{p} \cdot V \subseteq U$. Pick $\vec{x} \in U \cap E_{k}$ with $\vec{x} \in I_{k}$ if $V \cap I_{k} \neq \varnothing$, in particular if $\vec{q} \in I$. If $\vec{x} \in I_{k}$ pick $a_{1}, a_{2}, \ldots, a_{n+1}$, $m(1), m(2), \ldots, m(n)$, and $F_{1}, F_{2}, \ldots, F_{n+1}$ as guaranteed by the definition of $I_{k}$ and let $u=\max F_{n+1}+1$. If $x \notin I_{k}$ pick $a \in P_{k}$ with $\vec{x}=(a, a, \ldots, a)$ and pick $F \in \mathscr{S}(a)$ with $\min F \geq k$ and let $u=\max F+1$.

Now $\vec{p} \cdot \vec{x} \in U$ and $\vec{x} \in S^{l+1}$ so that $\rho_{\vec{x}}$ is continuous. Pick a neighborhood $W$ of $\vec{p}$ with $W \cdot \vec{x} \subseteq U$ and pick $\vec{z} \in W \cap E_{u}$ with $\vec{z} \in I_{u}$ if $W \cap I_{u} \neq \varnothing$, in particular if $\vec{p} \in I$. If $z \in I_{u}$ pick $b_{1}, b_{2}, \ldots, b_{t+1}, r(1), r(2), \ldots, r(t)$, and $G_{1}, G_{2}, \ldots, G_{t+1}$ as guaranteed by the definition of $I_{u}$. (That is $\vec{z}=$ $\left(b_{t+1} b_{t} \cdots b_{1}, b_{t+1} y_{1, r(t)} b_{t} \cdots b_{2} y_{1, r(1)} b_{1}, \ldots, b_{t+1} y_{l, r(t)} b_{t} \cdots b_{2} y_{l, r(1)} b_{1}\right)$, each $b_{j} \in P_{1}$ and each $G_{j} \in \mathscr{S}\left(b_{j}\right)$ and $\min G_{t+1}>r(t)>\max G_{t} \geq \cdots>$ $\max G_{2} \geq \min G_{2}>r(1)>\max G_{1} \geq \min G_{1} \geq u$.) If $\vec{z} \in I_{u}$ pick $b \in P_{u}$ with $\vec{z}=(b, b, \ldots, b)$.

If $\vec{z} \in I_{u}$ and $\vec{x} \in I_{k}$ we have

$$
\begin{aligned}
& \vec{z} \cdot \vec{x}=\left(b_{t+1} b_{t} \cdots b_{1} a_{n+1} a_{n} \cdots a_{1}, b_{t+1} y_{1, r(t)} b_{t}\right. \\
& \cdots b_{2} y_{1, r(1)} b_{1} a_{n+1} y_{1, m(n)} a_{n} \cdots a_{2} y_{1, m(1)} a_{1}, \ldots, \\
&\left.b_{t+1} y_{l, r(t)} b_{t} \cdots b_{2} y_{l, r(1)} b_{1} a_{n+1} y_{l, m(n)} a_{n} \cdots a_{2} y_{l, m(1)} a_{1}\right) .
\end{aligned}
$$

Further, if $j \in\{2,3, \ldots, t+1\}$ we have $b_{j} \in P_{1}$ and $G_{j} \in \mathscr{S}\left(b_{j}\right)$; if $j \in$ $\{1,2, \ldots, n\}$ we have $a_{j} \in P_{1}$ and $F_{j} \in \mathscr{S}\left(a_{j}\right)$; also $b_{1} a_{n+1} \in P_{1}$ and $G_{1} \cup$ $F_{n+1} \in \mathscr{S}\left(b_{1} a_{n+1}\right)$. Since $\min G_{t+1}>r(t)>\cdots>r(1)>\max \left(G_{1} \cup F_{n+1}\right)>$ $\min \left(G_{1} \cup F_{n+1}\right)>m(n)>\cdots>m(1)>\max F_{1} \geq \min F_{1} \geq k, \vec{z} \cdot \vec{x} \in I_{k}$.

If $z \in I_{u}$ and $x \notin I_{k}$ we have $\vec{z} \cdot \vec{x}=\left(b_{t+1} b_{t} \cdots b_{1} a, b_{t+1} y_{1, r(t)} b_{t} \cdots\right.$ $\left.b_{2} y_{1, r(1)} b_{1} a, \ldots, b_{t+1} y_{l, r(t)} b_{t} \cdots b_{2} y_{l, r(1)} b_{1} a\right)$ so that $\vec{z} \cdot \vec{x} \in I_{k}$.

If $\vec{z} \notin I_{u}$ and $\vec{x} \in I_{k}$ we have $\vec{z} \cdot \vec{x}=\left(b a_{n+1} a_{n} \cdots a_{1}, b a_{n+1} y_{1, m(n)} a_{n} \cdots\right.$ $\left.a_{2} y_{1, m(1)} a_{1}, \ldots, b a_{n+1} y_{l, m(a)} a_{n} \cdots a_{2} y_{l, m(1)} a_{1}\right)$ so that $\vec{z} \cdot \vec{x} \in I_{k}$.

If $\vec{z} \notin I_{u}$ and $\vec{x} \notin I_{k}$ we have $\vec{z} \cdot \vec{x}=(b a, b a, \ldots, b a)$ and $b a \in P_{k}$ so $\vec{z} \cdot \vec{x} \in E_{k}$.

The following theorem and its consequence Corollary 2.7 (while not the major results of this section) are already strong enough for many of our applications, including the noncommutative van der Waerden theorem. In fact all of the results of the next section except for Corollary 3.7 depend only on these results.

2.6. Theorem. Let $S$ be a semigroup, let $l \in \mathbb{N}$, and for each $i \in\{0,1, \ldots, l\}$ let $\left\langle y_{i, n}\right\rangle_{n=1}^{\infty}$ be a sequence in $S$. Let $X$ and $P_{k}$ be as in Definition 2.2. Let $R$ be a minimal right ideal of $X$, let $p \in R$, and let $A \in p$. For each $k \in \mathbb{N}$ there exist $n \in \mathbb{N}, a_{1}, a_{2}, \ldots, a_{n+1} \in P_{k}, m(1), m(2), \ldots, m(n) \in \mathbb{N}$ and $F_{1}, F_{2}, \ldots, F_{n+1} \subseteq \mathbb{N}$ with each $F_{j} \in \mathscr{S}\left(a_{j}\right)$ and $\min F_{n+1}>m(n)>$ $\max F_{n} \geq \min F_{n}>m(n-1)>\cdots>m(1)>\max F_{1} \geq \min F_{1} \geq k$ and 
$\left\{a_{n+1} a_{n} \cdots a_{1}, a_{n+1} y_{1, m(n)} a_{n} \cdots a_{2} y_{1, m(1)} a_{1}, \ldots, a_{n+1} y_{l, m(n)} a_{n} \cdots a_{2} y_{l, m(1)} a_{1}\right\}$ $\subseteq A$.

Proof. Let $\vec{p}=(p, p, \ldots, p)$. We first show $\vec{p} \in E$. To this end let $t \in \mathbb{N}$ and let $U$ be a neighborhood of $\vec{p}$ in $T$. Pick $A_{0}, A_{1}, \ldots, A_{l} \in p$ with $\bar{A}_{0} \times \bar{A}_{1} \times \cdots \times \bar{A}_{l} \subseteq U$. Since $p \in X \subseteq \bar{P}_{t}$, pick $a \in \bigcap_{i=0}^{l} A_{i} \cap P_{t}$. Then

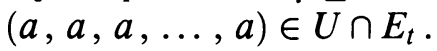

Now $E$ is compact and $\vec{p} \cdot E$ is a right ideal of $E$ so pick a minimal right ideal $R^{*}$ of $E$ with $R^{*} \subseteq \vec{p} \cdot E$. By [5, Corollary 2.10] any compact left topological semigroup has an idempotent so pick $\vec{q} \in R^{*}$ with $\vec{q} \cdot \vec{q}=\vec{q}$. Since $\vec{q} \in \vec{p} \cdot E$ pick $\vec{r} \in E$ with $\vec{q}=\vec{p} \cdot \vec{r}$.

We show now that $\vec{q} \cdot \vec{p}=\vec{p}$. Let $i \in\{0,1, \ldots, l\}$. We show $q_{i} \cdot p=p$. We have $q_{i}=p \cdot r_{i} \in R \cdot X \subseteq R$. (By Lemma 2.4 each $r_{i} \in X$.) Thus $q_{i} \cdot X$ is a right ideal of $X$ contained in $R$ so $q_{i} \cdot X=R$. Pick $t_{i} \in X$ with $q_{i} \cdot t_{i}=p$. Then $q_{i} \cdot p=q_{i} \cdot q_{i} \cdot t_{i}=q_{i} \cdot t_{i}=p$ as required.

Since $\vec{q} \cdot \vec{p}=\vec{p}$ we have $\vec{p} \in R^{*}$. Since $I$ is an ideal of $E, R^{*} \subseteq I$. (The intersection is nonempty since $I$ is a left ideal. Thus $R^{*} \cap I$ is a right ideal contained in $R^{*}$ so $R^{*} \cap I=R^{*}$.) Since $\vec{p} \in I \subseteq \mathrm{cl}_{T} I_{k}$ and $\bar{A} \times \bar{A} \times \cdots \times \bar{A}$ is a neighborhood of $\vec{p}$ we may pick $\vec{x} \in(\bar{A} \times \bar{A} \times \cdots \times \bar{A}) \cap I_{k}$. Since $\vec{x} \in I_{k}$, there exist $n \in \mathbb{N}, a_{1}, a_{2}, \ldots, a_{n+1} \in P_{k}, m(1), m(2), \ldots, m(n) \in \mathbb{N}$, and $F_{1}, F_{2}, \ldots, F_{n+1} \subseteq \mathbb{N}$ with each $F_{j} \in \mathscr{S}\left(a_{j}\right)$ and $\min F_{n+1}>m(n)>$ $\max F_{n}>\cdots>m(1)>\max F_{1} \geq \min F_{1} \geq k$ and $\vec{x}=\left(a_{n+1} a_{n} \cdots a_{1}\right.$, $\left.a_{n+1} y_{1, m(n)} a_{n} \cdots a_{2} y_{1, m(1)} a_{1}, \ldots, a_{n+1} y_{l, m(n)} a_{n} \cdots a_{2} y_{l, m(1)} a_{1}\right)$.

The next corollary is derivable from Theorem $2: 6$ in the same way that Theorem 2.12 is derived from Theorem 2.8. Since it is in fact a special case of Theorem 2.12 we do not present its proof here. It is stated separately because it is used in our simpler applications and is not as complicated as Theorem 2.12.

2.7. Corollary. Let $S$ be a cancellative semigroup with a 1 , let $l \in \mathbb{N}$, and let $\left\langle y_{i, n}\right\rangle_{n=1}^{\infty}$ for $i \in\{1,2, \ldots, l\}$ be one-to-one sequences in $S \backslash\{1\}$. Assume that for $c, d \in S$ and $i \neq j$ in $\{1,2, \ldots, l\},\left\{n \in \mathbb{N}: y_{i, n} \cdot c=y_{j, n} \cdot d\right\}$ is finite. Let $r \in \mathbb{N}$ and let $S=\bigcup_{k=1}^{r} A_{k}$. There exists $k \in\{1,2, \ldots, r\}, n \in \mathbb{N}$ $m(1)<m(2)<\cdots<m(n)$ in $\mathbb{N}$ and $a_{1}, a_{2}, \ldots, a_{n+1}$ in $S$ with the set $\left\{a_{n+1} a_{n} \cdots a_{1}, a_{n+1} y_{1, m(n)} a_{n} \cdots a_{2} y_{1, m(1)} a_{1}, \ldots, a_{n+1} y_{l, m(n)} a_{n} \cdots a_{2} y_{l, m(1)} a_{1}\right\}$ contained in $A_{k}$ and consisting of $l+1$ distinct elements.

The following theorem and its consequence, Theorem 2.12, are the major results of this section.

2.8. Theorem. Let $S$ be a semigroup, let $l \in \mathbb{N}$, and for each $i \in\{0,1, \ldots, l\}$ let $\left\langle y_{i, n}\right\rangle_{n=1}^{\infty}$ be a sequence in $S$. Let $X, P_{k}$, and $\mathscr{S}$ be as in Definition 2.2 . Let $R$ be a minimal right ideal of $X$, let $p$ be an idempotent in $R$, and let $A \in$ $p$. There exist sequences $\langle n(t)\rangle_{t=1}^{\infty}$ and $\left\langle\langle m(t, j)\rangle_{j=1}^{n(t)}\right\rangle_{t=1}^{\infty}$ in $\mathbb{N}$ and sequences $\left\langle\left\langle a_{t, j}\right\rangle_{j=1}^{n(t)+1}\right\rangle_{t=1}^{\infty}$ and $\left\langle\langle z(t, i)\rangle_{i=1}^{l}\right\rangle_{t=1}^{\infty}$ in $S$ and a sequence $\left\langle\left\langle F_{t, j}\right\rangle_{j=1}^{n(t)+1}\right\rangle_{t=1}^{\infty}$ of finite nonempty subsets of $\mathbb{N}$ satisfying:

(1) For $t \in \mathbb{N}$ and $j \in\{1,2, \ldots, n(t)+1\}, a_{t, j} \in P_{1}$ and $F_{t, j} \in \mathscr{S}\left(a_{t, j}\right)$;

(2) For $t \in \mathbb{N}, \min F_{t, n(t)+1}>m(t, n(t))>\max F_{t, n(t)} \geq \min F_{t, n(t)}>\cdots>$ $m(t, 1)<\max F_{t, 1}$;

(3) For $t \in \mathbb{N}, \min F_{t+1,1}>\max F_{t, n(t)+1}$; 
(4) For $t \in \mathbb{N}$ and $i \in\{1,2, \ldots, l\}, z(t, i)=a_{t, n(t)+1} y_{i, m(t, n(t))} a_{t, n(t)} \cdots$ $a_{t, 2} y_{i, m(t, 1)} a_{t, 1}$;

(5) For $t \in \mathbb{N}, z(t, 0)=a_{t, n(t+1)} a_{t, n(t)} \cdots a_{t, 1}$;

(6) Given a finite nonempty $F \subseteq \mathbb{N}$ and $f: F \rightarrow\{0,1, \ldots, l\}$ one has $\prod_{t \in F} z(t, f(t)) \in A$, where the product is in decreasing order of indices.

Proof. Let $A_{1}=A$ and let $B_{1}=\left\{x \in A_{1}: A_{1} / x \in p\right\}$. Since $p \cdot p=p$ we have $B_{1} \in p$. Choose $n(1) \in \mathbb{N}, a_{1,1}, a_{1,2}, \ldots, a_{1, n(1)+1} \in p_{1}, m(1,1)$, $m(1,2), \ldots, m(1, n(1)) \in \mathbb{N}$ and $F_{1,1}, F_{1,2}, \ldots, F_{1, n(1)+1} \subseteq \mathbb{N}$ as guaranteed by Theorem 2.6 for $B_{1}$. Define $z(1, i)$ for $i \in\{0,1, \ldots, l\}$ as required by conclusions (4) and (5). Then $\{z(1,0), z(1,1), \ldots, z(1, l)\} \subseteq B_{1}$. Let $A_{2}=A_{1} \cap \bigcap_{i=0}^{l}\left(A_{1} / z(1, i)\right)$. Then $A_{2} \in p$.

Inductively given $A_{t} \in p$, let $B_{t}=\left\{x \in A_{t}: A_{t} / x \in p\right\}$. Letting $k=$ $\max F_{t-1, n(t-1)+1}+1$, choose $n(t) \in \mathbb{N}, a_{t, 1}, a_{t, 2}, \ldots, a_{t, n(t)+1} \in P_{k}, m(t, 1)$, $m(t, 2), \ldots, m(t, n(t)) \in \mathbb{N}$ and $F_{t, 1}, F_{t, 2}, \ldots, F_{t, n(t)+1} \subseteq \mathbb{N}$ with $\min F_{t, 1} \geq$ $k$ as guaranteed by Theorem 2.6 for $B_{t}$. For $i \in\{0,1, \ldots, l\}$ define $z(t, i)$ as required by conclusions (4) and (5). Then $\{z(t, 0), z(t, 1), \ldots, z(t, l)\} \subseteq$ $B_{t}$. Let $A_{t+1}=A_{t} \cap \bigcap_{i=0}^{l}\left(A_{t} / z(t, i)\right)$. Then $A_{t+1} \in p$.

By the construction we have that for all $t \in \mathbb{N}$, conclusions (1), (2), (3), (4) and (5) hold. We verify (6) by proving by induction on $|F|$ that if $r=\min F$, then $\prod_{t \in F} z(t, f(t)) \in A_{r}$. If $|F|=1$, we have $\prod_{t \in F} z(t, f(t))=z(r, f(r)) \in$ $B_{r} \subseteq A_{r}$. Assume $|F|>1$, let $G=F \backslash\{r\}$ and let $u=\min G$. Then by our induction hypothesis $\prod_{t \in G} z(t, f(t)) \in A_{u} \subseteq A_{r+1} \subseteq A_{r} / z(r, f(r))$. Thus $\prod_{t \in F} z(t, f(t))=\left(\prod_{t \in G} z(t, f(t))\right) \cdot z(r, f(r)) \in A_{r}$.

2.9. Definition. A subset $A$ of $S$ is central if and only if there exist a minimal right ideal $R$ of $\beta S$ and an idempotent $p \in R$ with $A \in p$.

We introduced the above notion of central in [2], using that name because we could prove a generalization of [6, Proposition 8.21], a result about central subsets of $\mathbb{N}$ defined dynamically. The same definition could be applied in an arbitrary semigroup. With the help of B. Weiss we were able to show [2, Corollary 6.12] that, at least for countable semigroups, the two notions of central in fact coincide. (This result had been anticipated by S. Glasner who showed essentially the same thing in case $S$ is an abelian group [8, Proposition 4.6].)

The following corollary generalizes Theorem 4.11 of [2] where the result was restricted to certain commutative semigroups which were called prenatural. The special case was already strong enough to yield several results, including for example Deuber's $(m, p, c)$-set Theorem [4].

In the statement of Corollary 2.10 we put the word countable in parentheses because it is needed for the proof which we present here but is not needed for the conclusion to hold. (To prove the result for an arbitrary commutative semigroup one removes the restriction on the $a$ 's in the definition of $E_{k}$ and $I_{k}$ and replaces $X$ by $\beta S$.)

2.10. Corollary. Assume $S$ is commutative $C$ (and countable), let $A$ be central in $S$, let $l \in \mathbb{N}$, and let for each $i \in\{1,2, \ldots, l\},\left\langle y_{i, n}\right\rangle_{n=1}^{\infty}$ be a sequence in $S$. There exist sequences $\left\langle a_{t}\right\rangle_{t=1}^{\infty}$ and $\left\langle\langle z(t, i)\rangle_{i=0}^{l}\right\rangle_{t=1}^{\infty}$ and a sequence $\left\langle H_{t}\right\rangle_{t=1}^{\infty}$ of pairwise disjoint finite nonempty subsets of $\mathbb{N}$ such that

(1) For $t \in \mathbb{N}, z(t, 0)=a_{t}$;

(2) For $t \in \mathbb{N}$ and $i \in\{1,2, \ldots, l\}, z(t, i)=a_{t} \cdot \prod_{m \in H_{t}} y_{i, m}$; 
(3) Whenever $F$ is a finite nonempty subset of $\mathbb{N}$ and $f: F \rightarrow\{0,1, \ldots, l\}$, one has $\prod_{t \in F} z(t, f(t)) \in A$.

Proof. Let $\left\langle y_{0, n}\right\rangle_{n=1}^{\infty}$ be any sequence in $S$ which takes on each value in $S$ infinitely often. Then each $P_{k}=S$ so $X=\beta S$. Since $A$ is central in $S$, pick a minimal right ideal $\mathbb{R}$ of $\beta S=X$ and pick an idempotent $p \in R$ with $A \in p$. Then Theorem 2.8 applies. For each $t \in \mathbb{N}$, let $a_{t}=a_{t, n(t)+1} a_{t, n(t)} \cdots a_{t, 1}$ and let $H_{t}=\{m(t, 1), m(t, 2), \ldots, m(t, n(t))\}$.

It is of course possible that our efforts to avoid triviality in our conclusions are doomed. As an extreme example, consider a trivial semigroup $S$ where, for some fixed $a \in S, x \cdot y=a$ for all $x$ and $y$. As a less trivial example consider a left-zero semigroup (that is one in which $x \cdot y=x$ for all $x, y \in S$ ). In this case each of the terms produced by Theorem 2.6 is just $a_{n+1}$. We conclude this section by determining a simple condition on $S$ and the sequences $\left\langle y_{i, n}\right\rangle_{n=1}^{\infty}$ for $i \in\{1,2, \ldots, l\}$ which guarantees that the results of Theorems 2.6 and 2.8 are not trivial.

2.11. Lemma. Let $S$ be a cancellative semigroup with a 1 and let for $i \in$ $\{1,2, \ldots, l\}\left\langle y_{i, n}\right\rangle_{n=1}^{\infty}$ be one-to-one sequences in $S \backslash\{1\}$. Assume that for $c, d$ $\in S$ and $i \neq j$ in $\{1,2, \ldots, l\} \quad\left\{n \in \mathbb{N}: y_{i, n} \cdot c=y_{j, n} \cdot d\right\}$ is finite. Then there is an increasing sequence $\langle k(n)\rangle_{n=1}^{\infty}$ in $\mathbb{N}$ such that whenever $F$ and $G$ are finite subsets of $\mathbb{N}$ and $f: F \rightarrow\{1,2, \ldots, l\}$ and $g: G \rightarrow\{1,2, \ldots, l\}$ and $\prod_{n \in F} y_{f(n), k(n)}=\prod_{n \in G} y_{g(n), k(n)}$ one has $F=G$ and $f=g$. (Here the products are in decreasing order of indices and $\prod_{n \in \varnothing} y_{f(n), k(n)}=1$.)

Proof. We have (with $c=d=1$ ) that for each $i \neq j$ in $\{1,2, \ldots, l\}$, $\left\{n \in \mathbb{N}: y_{i, n}=y_{j, n}\right\}$ is finite so pick $k(1)$ with $y_{i, k(1)} \neq y_{j, k(1)}$ for $i \neq j$.

Let $t>1$ be given and assume we have chosen $k(1), k(2), \ldots, k(t-1)$. Let $H_{t}=\left\{\prod_{n \in F} y_{f(n), k(n)}: F \subseteq\{1,2, \ldots, t-1\}\right.$ and $\left.f: F \rightarrow\{1,2, \ldots, l\}\right\}$. (Note $F=\varnothing$ is allowed so $1 \in H_{t}$.) Let $B=\{n \in \mathbb{N}$ : for some $i \in\{1,2, \ldots, l\}$, $\left.\left(y_{i, n} \cdot H_{t}\right) \cap H_{t} \neq \varnothing\right\}$. Now given $i \in\{1,2, \ldots, l\}$ and $a, b \in H_{t}$ we have, by right cancellation and the fact that $\left\langle y_{i, n}\right\rangle_{n=1}^{\infty}$ is one-to-one, that at most one $n$ has $y_{i, n} \cdot a=b$. Consequently $B$ is finite.

Now given $c, d \in H_{t}$ and $i \neq j$ in $\{1,2, \ldots, l\}$, let $D(c, d, i, j)=$ $\left\{n \in \mathbb{N}: y_{i, n} \cdot c=y_{j, n} \cdot d\right\}$. By assumption each $D(c, d, i, j)$ is finite. Pick $k(t)>k(t-1)$ with $k(t) \in \mathbb{N} \backslash\left(B \cup \bigcup\left\{D(c, d, i, j): c, d \in H_{t}\right.\right.$, and $i, j \in$ $\{1,2, \ldots, l\}$ with $i \neq j\})$.

Suppose now that the conclusion fails and pick $F, G, f$, and $g$ with $\prod_{n \in F} y_{f(n), k(n)}=\prod_{n \in G} y_{g(n), k(n)}, f \neq g$, and $\max (F \cup G)$ smallest possible among all such counterexamples. (Note $F \cup G \neq \varnothing$ since the only function with empty domain is the empty function.) Let $t=\max (F \cup G)$ and assume without loss of generality that $t \in F$. We claim that also $t \in G$. Otherwise let $F^{\prime}=F \backslash\{t\}$ and let $c=\prod_{n \in F^{\prime}} y_{f(n), k(n)}$ and $d=\prod_{n \in G} y_{g(n), k(n)}$. Then $c, d \in H_{t}$ and $y_{f(t), k(t)} \cdot c=d$ so $k(t) \in B$, a contradiction.

Thus $t \in G$. Let $F^{\prime}=F \backslash\{t\}$ and $G^{\prime}=G \backslash\{t\}$. Let $c=\prod_{n \in F^{\prime}} y_{f(n), k(n)}$ and let $d=\prod_{n \in G^{\prime}} y_{g(n), k(n)}$. Then $c, d \in H_{t}$ and $y_{f(t), k(t)} \cdot c=y_{g(t), k(t)} \cdot d$. If $f(t) \neq g(t)$ we would have $k(t) \in D(c, d, f(t), g(t))$, a contradiction. Thus $f(t)=g(t)$. But then $\left.f\right|_{F^{\prime}} \neq\left. g\right|_{G^{\prime}}$ so we have a counterexample with $\max \left(F^{\prime} \cup G^{\prime}\right)<t$, a contradiction. 
We can now demonstrate that in very common situations we are guaranteed the nontriviality of our results. Observe that if $S$ is cancellative but does not have a 1 , one may add a 1 keeping $S \cup\{1\}$ cancellative provided that for all $x$ and $y \in S$ one has $x \neq x y$ and $y \neq x y$.

2.12. Theorem. Let $S$ be a cancellative semigroup with a 1 , let $l \in \mathbb{N}$, and let for $i \in\{1,2, \ldots, l\}\left\langle y_{i, n}\right\rangle_{n=1}^{\infty}$ be one-to-one sequences in $S \backslash\{1\}$. Assume that for $c, d \in S$ and $i \neq j$ in $\{1,2, \ldots, l\},\left\{n \in \mathbb{N}: y_{i, n} \cdot c=y_{j, n} \cdot d\right\}$ is finite. Let $r \in \mathbb{N}$ and let $S=\bigcup_{u=1}^{r} A_{u}$. There exist $u \in\{1,2, \ldots, r\}$, sequences $\langle n(t)\rangle_{t=1}^{\infty}$ and $\left\langle\langle m(t, j)\rangle_{j=1}^{n(t)}\right\rangle_{t=1}^{\infty}$ in $\mathbb{N}$, sequences $\left\langle\left\langle a_{t, j}\right\rangle_{j=1}^{n(t)}\right\rangle_{t=1}^{\infty}$ and $\left\langle\langle z(t, i)\rangle_{i=1}^{l}\right\rangle_{t=1}^{\infty}$ in $S$ and a sequence $\left\langle\left\langle F_{t, j}\right\rangle_{j=1}^{n(t)+1}\right\rangle_{t=1}^{\infty}$ of finite nonempty subsets of $\mathbb{N}$ satisfying:

(1) For $t \in \mathbb{N}$ and $j \in\{1,2, \ldots, n(t)+1\}$ there is a function $f: F_{t, j} \rightarrow$ $\{1,2, \ldots, l\}$ such that $a_{t, j}=\prod_{b \in F_{t, j}} y_{f(b), b}$

(2) For $t \in \mathbb{N}, \min F_{t, n(t)+1}>m(t, n(t))>\max F_{t, n(t)} \geq \min F_{t, n(t)}>\cdots>$ $m(t, 1)>\max F_{t, 1}$;

(3) For $t \in \mathbb{N}, \min F_{t+1,1}>\max F_{t, n(t)+1}$;

(4) For $t \in \mathbb{N}$ and $i \in\{1,2, \ldots, l\}, z(t, i)=a_{t, n(t)+1} y_{i, m(t, n(t))} a_{t, n(t)} \cdots$ $a_{t, 2} y_{i, m(t, 1)} a_{t, 1}$

(5) For $t \in \mathbb{N}, z(t, 0)=a_{t, n(t+1)} a_{t, n(t)} \cdots a_{t, 1}$;

(6) Given a finite nonempty $F \subseteq \mathbb{N}$ and $f: F \rightarrow\{0,1, \ldots, l\}$ one has $\prod_{t \in F} z(t, f(t)) \in A_{u} ;$ and

(7) Given finite nonempty $F, G \subseteq \mathbb{N}, f: F \rightarrow\{0,1, \ldots, l\}$, and $g: G \rightarrow$ $\{0,1, \ldots, l\}$, if $(F, f) \neq(G, g)$, then $\prod_{t \in F} z(t, f(t)) \neq \prod_{t \in G} z(t, g(t))$.

Proof. Choose a sequence $\langle k(n)\rangle_{n=1}^{\infty}$ as guaranteed by Lemma 2.11 and for each $n \in \mathbb{N}$ and each $i \in\{1,2, \ldots, l\}$, let $y_{i, n}^{*}=y_{i, k(n)}$. Also for $n \in \mathbb{N}$, let $y_{0, n}^{*}=y_{1, n}^{*}$. Let $P_{k}, \mathscr{S}(a)$ and $X$ be defined as in Definition 2.2 for the sequences $\left\langle\left\langle y_{i, n}^{*}\right\rangle_{n=1}^{\infty}\right\rangle_{i=0}^{l}$. Pick a minimal right ideal $R$ of $X$ and (again by [5, Corollary 2.10]) pick an idempotent $p$ in $R$. Pick $u \in\{1,2, \ldots, r\}$ with $A_{u} \in p$.

Choose sequences

$$
\begin{gathered}
\langle n(t)\rangle_{n=1}^{\infty}, \quad\left\langle\left\langle m^{*}(t, j)\right\rangle_{j=1}^{n(t)}\right\rangle_{t=1}^{\infty}, \quad\left\langle\left\langle a_{t, j}\right\rangle_{j=1}^{n(t)+1}\right\rangle_{t=1}^{\infty}, \\
\left\langle\langle z(t, i)\rangle_{i=0}^{l}\right\rangle_{t=1}^{\infty}, \quad \text { and }\left\langle\left\langle F_{t, j}^{*}\right\rangle_{j=1}^{n(t)+1}\right\rangle_{t=1}^{\infty}
\end{gathered}
$$

as guaranteed by Theorem 2.8. For $t \in \mathbb{N}$ and $j \in\{1,2, \ldots, n(t)\}$ let $m(t, j)=k\left(m^{*}(t, j)\right)$. For $t \in \mathbb{N}$ and $j \in\{1,2, \ldots, n(t)+1\}$ let $F_{t, j}=$ $\left\{k(b): b \in F_{t, j}^{*}\right\}$. We show that all of the conclusions hold.

To verify (1), let $t \in \mathbb{N}$ and $j \in\{1,2, \ldots, n(t)+1\}$. Since $a_{t, j} \in P_{1}$ and $F_{t, j}^{*} \in \mathscr{S}\left(a_{t, j}\right)$ pick a function $h: F_{t, j}^{*} \rightarrow\{0,1, \ldots, l\}$ with $a_{t, j}=$ $\prod_{b \in F_{t, j}^{*}} y_{h(0), b}^{*}$. Since each $y_{0, b}^{*}=y_{1, b}^{*}$ we may in fact presume $0 \notin h\left[F_{t, j}\right]$. Define $f: F_{t, j} \rightarrow\{1,2, \ldots, l\}$ by $f(k(b))=h(b)$. Then

$$
\prod_{b \in F_{t, j}} y_{f(b), b}=\prod_{b \in F_{i, j}^{*}} y_{f(k(b)), k(b)}=\prod_{b \in F_{i, j}^{*}} y_{h(b), b}^{*} .
$$

Since, given $t$ and $j, F_{t, j}=k\left[F_{t, j}^{*}\right]$ and $m(t, j)=k\left(m^{*}(t, j)\right)$ conclusions (2), (3), and (4) follow immediately from the corresponding conclusions of Theorem 2.8. 
Conclusions (5) and (6) are unchanged from the corresponding conclusions of Theorem 2.8 .

Finally to verify conclusion (7), although the argument is reasonably simple, we will need to set up some notation. Let $F, G, f$, and $g$ be given as there. For each $t \in F \cup G$ and each $j \in\{1,2, \ldots, n(t)+1\}$, pick $h(t, j): F_{t, j}^{*} \rightarrow\{1,2, \ldots, l\}$ with $a_{t, j}=\prod_{c \in F_{t, j}^{*}} y_{h(t, j)(c), c}^{*}$. For each $t \in F$, if $f(t) \neq 0$ let $L_{t}=\left\{m^{*}(t, 1), m^{*}(t, 2), \ldots, m^{*}(t, n(t))\right\} \cup \bigcup_{j=1}^{n(t)+1} F_{t, j}^{*}$. If $f(t)=0$ let $L_{t}=\bigcup_{j=1}^{n(t)+1} F_{t, j}^{*}$. For $t \in G$, if $g(t) \neq 0$ let $M_{t}=\left\{m^{*}(t, 1)\right.$, $\left.m^{*}(t, 2), \ldots, m^{*}(t, n(t))\right\} \cup \bigcup_{j=1}^{n(t)+1} F_{t, j}^{*}$. If $g(t)=0$, let $M_{t}=\bigcup_{j=1}^{n(t)+1} F_{t, j}^{*}$. Let $D=\bigcup_{t \in F} L_{t}$ and let $E=\bigcup_{t \in G} M_{t}$. Define $d: D \rightarrow\{1,2, \ldots, l\}$ as follows, observing that the $L_{t}$ 's are pairwise disjoint as are the sets displayed in the definition of each $L_{t}$. For $t \in F$ and $c \in F_{t, j}^{*}$ for some $j \in\{1,2, \ldots, n(t)+$ $1\}$ let $d(c)=h(t, j)(c)$. If $f(t) \neq 0$, let for $j \in\{1,2, \ldots, n(t)\}, d\left(m^{*}(t, j)\right)$ $=f(t)$. Likewise define $e: E \rightarrow\{1,2, \ldots, l\}$ by $e(c)=h(t, j)(c)$ for $c \in F_{t, j}^{*}$ and $e\left(m^{*}(t, j)\right)=g(t)$ when $g(t) \neq 0$.

Observe that, if $t \in F$ and $f(t)=0$, then $z(t, f(t))=\prod_{j=n(t)+1}^{1} a_{t, j}=$ $\prod_{j=n(t)+1}^{1} \prod_{c \in F_{t, j}^{*}} y_{h(t, j)(c), c}^{*}=\prod_{j=n(t)+1}^{1} \prod_{c \in F_{t, j}^{*}} y_{h(t, j)(c), k(c)}=\prod_{c \in L_{t}} y_{d(c), k(c)}$. Likewise if $t \in G$ and $g(t)=0, z(t, g(t))=\prod_{c \in M_{t}} y_{e(c), k(c)}$. Now let $t \in F$ with $f(t) \neq \varnothing$. Then

$$
\begin{aligned}
z(t, f(t)) & =a_{t, n(t)+1} \cdot \prod_{j=n(t)}^{1}\left(y_{f(t), m^{*}(t, j)}^{*} \cdot a_{t, j}\right) \\
& =\prod_{c \in F_{t, n(t)+1}^{*}} y_{h(t, n(t)+1)(c), c}^{*} \cdot \prod_{j=n(t)}^{1}\left(y_{f(t), m^{*}(t, j)}^{*} \cdot \prod_{c \in F_{t, j}^{*}} y_{h(t, j)(c), c}^{*}\right) \\
& =\prod_{c \in F_{t, n(t)+1}^{*}} y_{h(t, n(t)+1)(c), k(c)} \cdot \prod_{j=n(t)}^{1}\left(y_{f(t), k\left(m^{*}(t, j)\right)} \cdot \prod_{c \in F_{t, j}^{*}} y_{h(t, j)(c), k(c)}\right) \\
& =\prod_{c \in L_{t}} y_{d(c), k(c) .}
\end{aligned}
$$

Likewise if $t \in G$ and $g(t) \neq 0, z(t, g(t))=\prod_{c \in M_{t}} y_{e(c), k(c)}$. Suppose now that $\prod_{t \in F} z(t, f(t))=\prod_{t \in G} z(t, g(t))$. Then

$$
\begin{aligned}
\prod_{c \in D} y_{d(c), k(c)} & =\prod_{t \in F} \prod_{c \in L_{t}} y_{d(c), k(c)}=\prod_{t \in F} z(t, f(t))=\prod_{t \in G} z(t, g(t)) \\
& =\prod_{t \in G} \prod_{c \in M_{t}} y_{e(c), k(c)}=\prod_{c \in E} y_{e(c), k(c) .}
\end{aligned}
$$

Thus by Lemma $2.11 D=E$ and $d=e$. Now if $t \in F$ then $F_{t, 1}^{*} \subseteq D=E$ so $t \in G$ (by the disjointness conditions (2) and (3) of Theorem 2.8). Thus $F \subseteq G$ and likewise $G \subseteq F$. Since $(F, f) \neq(G, g)$ we must have $f \neq g$, so pick some $t \in F$ with $f(t) \neq g(t)$. Without loss of generality $f(t) \neq 0$. Then $m^{*}(t, 1) \in D=E$ so $g(t) \neq 0$. But then $d\left(m^{*}(t, 1)\right)=f(t) \neq g(t)=$ $e\left(m^{*}(t, 1)\right)$, a contradiction. 


\section{COMBINATORIAL CONSEQUENCES}

We present here several corollaries to the results of $\S 2$. All of these can be formulated like Theorems 2.8 and 2.12. That is the conclusion can be phrased as all decreasing products found by making choices from different $(l+1)$-tuples. However, except for Corollary 3.7, we choose to present the simpler (and we hope more comprehensible) versions which are phrased more like Theorem 2.6. Of these consequences Corollaries 3.1, 3.3, and 3.4 are new results.

3.1. Corollary (Noncommutative van der Waerden). Let $S$ be any semigroup, let $l, r \in \mathbb{N}$, let $\left\langle d_{m}\right\rangle_{m=1}^{\infty}$ be a sequence in $S$, and let $S=\bigcup_{k=1}^{r} A_{k}$. There exist $k \in\{1,2, \ldots, r\}, n \in \mathbb{N}, a_{1}, a_{2}, \ldots, a_{n+1} \in S$, and $m(1)<m(2)<\cdots<$ $m(n)$ in $\mathbb{N}$ with $\left\{a_{n+1} a_{n} \cdots a_{2} a_{1}, a_{n+1} d_{m(n)} a_{n} d_{m(n-1)} \cdots a_{2} d_{m(1)} a_{1}, a_{n+1} d_{m(n)}^{2} a_{n}\right.$ - $\left.d_{m(n-1)}^{2} \cdots a_{2} d_{m(1)}^{2} a_{2}, \ldots, a_{n+1} d_{m(n)}^{l} a_{n} d_{m(n-1)}^{l} \cdots a_{2} d_{m_{1}(1) a_{1}}^{l}\right\} \subseteq A_{k}$. If $S$ is cancellative with $a 1$ and for each $i \in\{1,2, \ldots, l\}$, the sequence $\left\langle d_{m}^{i}\right\rangle_{m=1}^{\infty}$ is one-to-one, then the $l+1$ entries can be chosen to be distinct.

Proof. For $i \in\{1,2, \ldots, l\}$ and $m \in \mathbb{N}$ let $y_{i, m}=d_{m}^{i}$ and let $y_{0, m}=d_{m}$ (or anything else you choose). Let $X$ be as in Definition 2.2 , let $R$ be a minimal right ideal of $X$, and pick $p \in R$. Pick $k \in\{1,2, \ldots, r\}$ such that $A_{k} \in p$ and apply Theorem 2.6 .

To see that the second conclusion holds we apply Corollary 2.7. For this we need only show that if $b, c \in S$ and $i \neq j$ in $\{1,2, \ldots, l\}$, then $\{m \in$ $\left.\mathbb{N}: d_{m}^{i} \cdot b=d_{m}^{j} \cdot c\right\}$ is finite. Assuming without loss of generality that $j<i$, let $m, n \in \mathbb{N}$ with $d_{m}^{i} \cdot b=d_{m}^{j} \cdot c$ and $d_{n}^{i} \cdot b=d_{n}^{j} \cdot c$. Then $d_{m}^{i-j} \cdot b=c=d_{n}^{i-j} \cdot b$ so $d_{m}^{i-j}=d_{n}^{i-j}$ so $m=n$.

3.2. Corollary (Commutative van der Waerden). Let $S$ be a commutative semigroup, let $l, r \in \mathbb{N}$, let $\left\langle d_{m}\right\rangle_{m=1}^{\infty}$ be a sequence in $S$, and let $S=\bigcup_{k=1}^{r} A_{k}$. There exist $k \in\{1,2, \ldots, r\}, a \in S$, and $d \in F P\left(\left\langle d_{m}\right\rangle_{m=1}^{\infty}\right)$ with $\{a$, ad, $\left.a d^{2}, \ldots, a d^{l}\right\} \subseteq A_{k}$.

Proof. Pick $k, n, a_{1}, a_{2}, \ldots, a_{n+1}$, and $m(1), m(2), \ldots, m(n)$ as guaranteed by Corollary 3.1. Let $a=a_{n+1} a_{n} \cdots a_{1}$ and $d=d_{m(n)} d_{m(n-1)} \cdots d_{1}$.

Of course if $S$ is $(\mathbb{N},+)$ Corollary 3.2 becomes Theorem 1.1 .

In connection with the following corollary, recall that it is known that for sufficiently large primes $p$ there are infinite noncommutative groups satisfying $x^{p}=e$ for all $x \in G$. (See [13].)

3.3. Corollary. Let $G$ be an infinite group, $p$ a prime, and assume for all $x \in G, x^{p}=e$. Let $\left\langle d_{m}\right\rangle_{m=1}^{\infty}$ be a one-to-one sequence in $G$. Let $r \in \mathbb{N}$ and let $G=\bigcup_{k=1}^{r} A_{k}$. There exist $k \in\{1,2, \ldots, r\}, n \in \mathbb{N}, a_{1}, a_{2}, \ldots, a_{n+1} \in G$, and $m(1)<m(2)<\cdots<m(n)$ in $\mathbb{N}$ with $B=\left\{a_{n+1} a_{n} \cdots a_{1}, a_{n+1} d_{m(n)} a_{n} \cdots\right.$ $\left.a_{2} d_{m(1)} a_{1}, a_{n+1} d_{m(n)}^{2} a_{n} \cdots a_{2} d_{m(1)}^{2} a_{1}, \ldots, a_{n+1} d_{m(n)}^{p-1} a_{n} \cdots a_{2} d_{m(1)}^{p-1} a_{1}\right\} \subseteq A_{k}$ and $|B|=p$.

Proof. By Corollary 3.1 we need only show that for each $i \in\{1,2, \ldots, p-1\}$ the sequence $\left\langle d_{m}^{i}\right\rangle_{m=1}^{\infty}$ is one-to-one. For this let $i \in\{1,2, \ldots, p-1\}$ and assume $m, n \in \mathbb{N}$ and $d_{m}^{i}=d_{n}^{i}$. Pick $t \in \mathbb{N}$ with $i \cdot t \equiv 1(\bmod p)$. Then $d_{m}=d_{m}^{i t}=d_{n}^{i t}=d_{n}$ so $m=n$.

Gallai's Theorem (from [14]) tells us, in its two-dimensional version, that if 
$\mathbb{N}^{2}$ is partitioned into finitely many classes and $l \in \mathbb{N}$ is given, then there are two length $l$ arithmetic progressions with the same increment whose cartesian product is contained in one class. We shall likewise state our results only in their two-dimensional versions. Passage to higher dimensions will pose no difficulty for the interested reader.

3.4. Corollary (Noncommutative Gallai). Let $S$ be any semigroup, let $l, r \in \mathbb{N}$, let $\left\langle d_{m}\right\rangle_{m=1}^{\infty}$ and $\left\langle e_{m}\right\rangle_{m=1}^{\infty}$ be sequences in $S$ and let $S \times S=\bigcup_{k=1}^{r} A_{k}$. There exist $k \in\{1,2, \ldots, r\}, n \in \mathbb{N}, a_{1}, a_{2}, \ldots, a_{n+1} \in S, b_{1}, b_{2}, \ldots, b_{n+1}$ $\in S$, and $m(1)<m(2)<\cdots<m(n)$ in $\mathbb{N}$ such that, if $C=\left\{a_{n+1} a_{n}\right.$ $\cdots a_{1}, a_{n+1} d_{m(n)} a_{n} \cdots a_{2} d_{m(1)} d_{1}, a_{n+1} d_{m(n)}^{2} a_{n} \cdots a_{2} d_{m(1)}^{2} a_{1}, \ldots, a_{n+1} d_{m(n)}^{l} a_{n} \cdots$ $\left.a_{2} d_{m(1)}^{l} a_{1}\right\}$ and $D=\left\{b_{n+1} b_{n} \cdots b_{1}, b_{n+1} e_{m(n)} b_{n} \cdots b_{2} e_{m(1)} b_{1}, b_{n+1} e_{m(n)}^{2} b_{n} \cdots\right.$ $\left.b_{2} e_{m(1)}^{2} a_{1}, \ldots, b_{n+1} e_{m(n)}^{l} b_{n} \cdots b_{2} e_{m(1)}^{l} b_{1}\right\}$ then $C \times D \subseteq A_{k}$. If $S$ is cancellative with $a 1$ and for each $i \in\{1,2, \ldots, l\}$ the sequences $\left\langle d_{m}^{i}\right\rangle_{m=1}^{\infty}$ and $\left\langle e_{m}^{i}\right\rangle_{m=1}^{\infty}$ are one-to-one, then we may conclude $|C \times D|=(l+1)^{2}$.

Proof. Let $S^{*}=S \times S$. For $i, j \in\{0,1, \ldots, l\}$ and $m \in \mathbb{N}$ let $\vec{y}_{i, j, m}=$ $\left(d_{m}^{i}, e_{m}^{j}\right)$ if $S$ has a 1 and otherwise let $\vec{y}_{i, j, m}=\left(d_{m}^{i+1}, e_{m}^{j+1}\right)$. Let $X$ be as in Definition 2.2 for $S^{*}$ and the $(l+1)^{2}$ sequences $\left\langle\vec{y}_{i, j, m}\right\rangle_{m=1}^{\infty}$ and an additional sequence $\left\langle\vec{y}_{0, m}\right\rangle_{m=1}^{\infty}$ with $\left.\vec{y}_{0, m}=\vec{y}_{1,1, m}\right)$. Pick a minimal right ideal $R$ of $X$, pick $p \in R$ and pick $k \in\{1,2, \ldots, r\}$ with $A_{k} \in p$. Applying Theorem 2.6 pick $n \in \mathbb{N},\left(x_{1}, z_{1}\right),\left(x_{2}, z_{2}\right), \ldots,\left(x_{n+1}, z_{n+1}\right) \in S^{*}$, and $m(1)<m(2)<\cdots<m(n)$ in $\mathbb{N}$ so that for each $i, j \in\{0,1, \ldots, l\}$, letting $\vec{w}_{i, j}=\left[\left(x_{n+1}, z_{n+1}\right) \vec{y}_{i, j, m(n)}\left(x_{n}, z_{n}\right) \cdots\left(x_{2}, z_{2}\right) \vec{y}_{i, j, m(1)}\left(x_{1}, z_{1}\right)\right]$, we have $\vec{w}_{i, j} \in A_{k}$. If $S$ has a 1 (so that $\vec{y}_{i, j, m}=\left(d_{m}^{i}, e_{m}^{j}\right)$ and in particular $\left.\vec{y}_{0,0, m}=(1,1)\right)$ let for each $t \in\{1,2, \ldots, n+1\}, a_{t}=x_{t}$ and $b_{t}=z_{t}$. Otherwise let $a_{n+1}=x_{n+1}, b_{n+1}=z_{n+1}$, and for $t \in\{1,2, \ldots, n\}$, let $a_{t}=d_{m(t)} x_{t}$ and $b_{t}=e_{m(t)} z_{t}$. Then in any case $\left(a_{n+1} a_{n} \cdots a_{1}, b_{n+1} b_{n} \cdots b_{1}\right)=\vec{w}_{0,0}$, for $j \in\{1,2, \ldots, l\},\left(a_{n+1} a_{n} \cdots a_{1}, b_{n+1} e_{m(n)}^{j} b_{n} \cdots b_{2} e_{m(1)}^{j} b_{1}\right)=\vec{w}_{0, j}$, for $i \in$ $\{1,2, \ldots, l\},\left(a_{n+1} d_{m(n)}^{i} a_{n} \cdots a_{2} d_{m(1)}^{i} a_{1}, b_{n+1} b_{n} \cdots b_{1}\right)=\vec{w}_{i, 0}$, and for $i, j \in$ $\{1,2, \ldots, l\},\left(a_{n+1} d_{m(n)}^{i} a_{n} \cdots a_{2} d_{m(1 j)}^{i} a_{1}, b_{n+1} e_{m(n)}^{j} b_{n} \cdots b_{2} e_{m(1)}^{j} b_{1}\right)=\vec{w}_{i, j}$.

To see that the second conclusion holds we apply Corollary 2.7 to the sequence $\left\langle\vec{y}_{i, j, m}\right\rangle_{m=1}^{\infty}$ with $(i, j) \neq(0,0)$. For this we need only show that if $i, j \in\{0,1, \ldots, l\}$ with $(i, j) \neq(0,0)$ one has $\left\langle\vec{y}_{i, j, m}\right\rangle_{m=1}^{\infty}$ is a one-toone sequence and that, given $(x, y)$ and $(u, v)$ in $S^{*}$ and $(i, j) \neq(a, \delta)$ in $(\{0,2, \ldots, l\} \times\{0,1, \ldots, l\}) \backslash\{(0,0)\}$, one has $\left\{m \in \mathbb{N}: \vec{y}_{i, j, m} \cdot(x, y)=\right.$ $\left.\vec{y}_{a, \delta, m} \cdot(u, v)\right\}$ is finite. That $\left\langle\vec{y}_{i, j, m}\right\rangle_{m=1}^{\infty}$ is one-to-one follows from the corresponding assumption about $\left\langle d_{m}^{i}\right\rangle_{m=1}^{\infty}$ and $\left\langle e_{m}^{j}\right\rangle_{m=1}^{\infty}$, since not both $i$ and $j$ are 0 . For the other assertion assume we have $m, n \in \mathbb{N}$ with $\vec{y}_{i, j, m} \cdot(x, y)=$ $\vec{y}_{a, \delta, m} \cdot(u, v)$ and $\vec{y}_{i, j, n} \cdot(x, y)=\vec{y}_{a, \delta, n} \cdot(u, v)$. Since $(i, j) \neq(a, \delta)$ we have without loss of generality that $i>a$. Then $d_{m}^{i} x=d_{m}^{a} u$ and $d_{n}^{i} x=d_{n}^{a} u$ so $d_{m}^{i-a} x=u=d_{n}^{i-a} x$ so $d_{m}^{i-a}=d_{n}^{i-a}$ so $m=n$.

Note that in Corollary 3.4 we may take $d_{m}=e_{m}$ and hence obtain the same increment. The reader is also invited to compose the two-dimensional analogue of Corollary 3.3.

We also obtain as corollaries some standard results.

3.5. Definition. Let $\Omega$ be a finite alphabet (i.e. set) and let $x$ be a variable not 
in $\Omega$. A variable word over $\Omega$ is a word over $\Omega \cup\{x\}$ in which $x$ actually occurs. If $\tau(x)$ is a variable word over $\Omega$ and $a \in \Omega$, then $\tau(a)$ is the word obtained by replacing all occurrences of $x$ by $a$.

Thus if $\Omega=\{a, b, c\}$ and $\tau(x)=a b x a x x b$, then $\tau(a)=a b a a a a b$ and $\tau(c)=a b c a c c b$.

3.6. Corollary (Hales-Jewett [10]). Let $\Omega$ be a finite alphabet, let $S$ be the set of (finite) words over $\Omega$, let $r \in \mathbb{N}$ and let $S=\bigcup_{k=1}^{r} A_{k}$. There exist $k \in$ $\{1,2, \ldots, r\}$ and a variable word $\tau(x)$ over $\Omega$ such that $\{\tau(b): b \in \Omega\} \subseteq A_{k}$. Proof. Let $\Omega=\left\{b_{1}, b_{2}, \ldots, b_{l}\right\}$. With concatenation as the operation, $S$ is a semigroup (the free semigroup over $\Omega$ ). For each $i \in\{1,2, \ldots, l\}$ and each $m \in \mathbb{N}$ let $y_{i, m}=b_{i}$, (and let $y_{0, m}=b_{1}$, say). Pick by Theorem $2.6 k \in$ $\{1,2, \ldots, r\}, n \in \mathbb{N}, a_{1}, a_{2}, \ldots, a_{n+1} \in S$ and $m(1)<m(2)<\cdots<m(n)$ in $\mathbb{N}$ with $\left\{a_{n+1} a_{n} \cdots a_{1}, a_{n+1} y_{1, m(n)} a_{n} \cdots a_{2} y_{1, m(1)} a_{1}, \ldots, a_{n+1} y_{l, m(n)} a_{n} \cdots\right.$ $\left.a_{2} y_{l, m(1)} a_{1}\right\} \subseteq A_{k}$. That is $\left\{a_{n+1} a_{n} \cdots a_{1}, a_{n+1} b_{1} a_{n} \cdots a_{2} b_{1} a_{1}, \ldots, a_{n+1} b_{l} a_{n} \ldots\right.$ $\left.a_{2} b_{l} a_{1}\right\} \subseteq A_{k}$. Then $\tau(x)=a_{n+1} x a_{n} x \cdots x a_{2} x a_{1}$ is as required.

Our final corollary is also the only one we present which uses most of the strength of Theorem 2.12. This corollary is a version of [3, Theorem 6.3].

3.7. Corollary. Let $\Omega$ be a finite alphabet, let $S$ be a set of finite words over $\Omega$, let $r \in \mathbb{N}$ and let $S=\bigcup_{k=1}^{r} A_{k}$. There exist $k \in\{1,2, \ldots, r\}$ and $a$ sequence $\left\langle\tau_{t}(x)\right\rangle_{t=1}^{\infty}$ of variable words over $\Omega$ such that, whenever $q \in \mathbb{N}$ and $f:\{1,2, \ldots, q\} \rightarrow \Omega$ one has $\tau_{q}(f(q)) \tau_{q-1}(f(q-1)) \cdots \tau_{2}(f(2)) \tau_{1}(f(1)) \in A_{k}$. Proof. Let $\Omega=\left\{b_{1}, b_{2}, \ldots, b_{l}\right\}$. For each $i \in\{1,2, \ldots, l\}$ and each $m$ $\in \mathbb{N}$, let $y_{i, m}=b_{i}$. Pick $k \in\{1,2, \ldots, r\}$ and sequences $\langle n(t)\rangle_{t=1}^{\infty}$, $\left\langle\langle m(t, j)\rangle_{j=1}^{n(t)}\right\rangle_{t=1}^{\infty},\left\langle\left\langle a_{t, j}\right\rangle_{j=1}^{n(t)+1}\right\rangle_{t=1}^{\infty}$, and $\left\langle\langle z(t, i)\rangle_{i=1}^{l}\right\rangle_{t=1}^{\infty}$ as guaranteed by Theorem 2.12 (replacing $u$ there by $k$ ). For each $t \in \mathbb{N}$, let $\tau_{t}(x)=a_{t, n(t)+1} x a_{t, n(t)} x$ $\cdots x a_{t, 2} x a_{t, 1}$. Then, since each $y_{i, m(t, s)}=b_{i}$ we have for each $i \in\{1,2, \ldots$, l\} that $z(t, i)=\tau_{t}\left(b_{i}\right)$. Given $q \in \mathbb{N}$, let $F=\{1,2, \ldots, q\}$. Given $f:\{1,2, \ldots, q\} \rightarrow \Omega$ define $g: F \rightarrow\{1,2, \ldots, l\}$ so that $f(t)=b_{g(t)}$. Then

$$
\begin{aligned}
& \tau_{q}(f(q)) \tau_{q-1}(f(q-1)) \cdots \tau_{1}(f(1)) \\
& \quad=z(q, g(q)) z(q-1, g(q-1)) \cdots z(1, g(1)) \\
& \quad=\prod_{t \in F} z(t, g(t)) \in A_{k} .
\end{aligned}
$$

We are grateful to Andreas Blass for pointing out that our Corollary 3.7 actually says something different than Theorem 6.3 of [3]. In the latter the variable words $\tau_{t}(x)$ for $t>1$ are required to have $x$ as their rightmost letter while in our construction this is strictly forbidden.

\section{REFERENCES}

1. V. Bergelson, H. Furstenberg, N. Hindman, and Y. Katznelson, An algebraic proof of van der Waerden's theorem, Enseign. Math. 35 (1989), 209-215.

2. V. Bergelson and N. Hindman, Nonmetrizable topological dynamics and Ramsey theory, Trans. Amer. Math. Soc. 320 (1990), 293-320.

3. T. Carlson and S. Simpson, A dual form of Ramsey's theorem, Adv. in Math. 53 (1984), 265-290.

4. W. Deuber, Partitionen und lineare Gleichungssysteme, Math. Z. 133 (1973), 109-123. 
5. R. Ellis, Lectures on topological dynamics, Benjamin, New York, 1969.

6. H. Furstenberg, Recurrence in ergodic theory and combinatorial number theory, Princeton Univ. Press, Princeton, N.J., 1981.

7. H. Furstenberg and B. Weiss, Topological dynamics and combinatorial number theory, J. Analyse Math. 34 (1978), 61-85.

8. S. Glasner, Divisible properties and the Stone-Čech compactification, Canad. J. Math. 32 (1980), 993-1007.

9. R. Graham, K. Leeb, and B. Rothschild, Ramsey's Theorem for a class of categories, Adv. in Math. 8 (1972), 417-433.

10. A. Hales and R. Jewett, Regularity and positional games, Trans. Amer. Math. Soc. 106 (1963), 222-229.

11. N. Hindman, Ultrafilters and combinatorial number theory, Number Theory Carbondale 1979 (M. Nathanson, ed.), Lecture Notes in Math., vol. 751, Springer-Verlag, Berlin and New York, 1979, pp. 119-184.

12. _ Ultrafilters and Ramsey Theory-an update, Set Theory and its Applications, (J. Steprans and S. Watson, eds.), Lecture Notes in Math., vol. 1401, Springer-Verlag, Berlin and New York, 1989, pp. 97-118.

13. A. Olshanskii, Groups of bounded period with subgroups of prime order, Algebra i Logika 21 (1982), 553-618 (Russian).

14. R. Rado, Studien zur Kombinatorik, Math. Z. 36 (1933), 242-280.

15. B. van der Waerden, Beweis einer Baudetschen Vermutung, Nieuw Arch. Wisk. 19 (1927), 212-216.

Department of Mathematics, Ohio State University, Columbus, Ohio 43210

Department of Mathematics, Howard University, Washington, D.C. 20059 